\title{
Factores climáticos, geográficos y fisiográficos que contribuyen a la distribución potencial del orégano (Lippia spp.) en México
}

\author{
Climatic, geographic and physiographic factors that contribute to the \\ distribution of oregano (Lippia spp.) in Mexico
}

\begin{abstract}
$\searrow$
Jesús Di Carlo Quiroz Velásquez', Martín Reyes Lara², Jesús Gerardo García Olivares', Ángel Salazar Bravo', Bianca Edith Bazán Cruz, José Luis Hernández Mendoza ${ }^{1 *}$

Quiroz Velásquez, J. D. C., Reyes Lara, M., García Olivares, J. G., Salazar Bravo, A., Bazán Cruz, B. E., Hernández Mendoza, J. L. Facłores climáticos, geográficos y fisiográficos que contribuyen a la distribución potencial del óregano (Lippia spp.) en México. Investigación y Ciencia de la Universidad Autónoma de Aguascalientes. Número 69: 21-25, septiembre-diciembre 2016.
\end{abstract}

\section{RESUMEN}

La presencia de Lippia graveolens es muy amplia, se distribuye en sitios diversos y confirma en algunos casos una asociación entre la presencia de la especie y factores climáticos, edáficos y geográficos. En Tamaulipas la humedad y la temperatura determinaron la distribución; en Durango, las características edáficas jugaron ese papel. En Yucatán los factores bioclimáticos no tienen influencia en la distribución de la especie, pero sí influyeron en los quimiotipos de la misma. En Zacatecas, Coahuila y Chihuahua, estados donde se recolecta más de $80 \%$ del orégano se carece de datos disponibles de condición y diversidad de esta especie. Con el programa Maxent versión 3.3.3k se realizó el análisis de la información y se estimó la distribución potencial de las tres especies de orégano, dos de las cuales son consideradas como sinónimos: Lippia graveolens y L. berlandieri.

Palabras clave: Lippia, orégano, predicción, ecología, Maxent, GBIF.

Keywords: Lippia, oregano, prediction, ecology, Maxent, GBIF.

Recibido: 19 de enero de 2015, aceptado: 21 de marzo de 2016

Laboratorio de Biotecnología Experimental, Centro de Biotecnología Genómica, Instituto Politécnico Nacional.

Instituto Tecnológico de Ciudad Victoria.

Unidad Académica Multidisciplinaria Reynosa.

Autor para correspondencia: jhernandezm@ipn.mx
ABSTRACT

The presence of Lippia graveolens is widely distributed in different sites in some cases confirming an association between the presence of the species and climate, soil and geographical factors. In the state of Tamaulipas, humidity and temperature determine the distribution; in Durango the soil characteristics play that role. In Yucatan, bioclimatic factors have no influence on the distribution of the species, but have an effect on the chemotypes. In the states of Zacatecas, Coahuila and Chihuahua, where more than $80 \%$ of oregano is collected, there is no data available on the condition and diversity of this species. Using the Maxent version 3.3.3k program an analysis of information was carried out to estimate the potential distribution of three species of oregano, two of which are considered synonymous: Lippia graveolens and L. berlandieri.

INTRODUCCIÓN

La distribución geográfica de Lippia graveolens va desde el sur de los Estados Unidos hasta Centroamérica y en especial en México tiene una amplia distribución, sobre todo en áreas serranas y desérticas, preferentemente con climas tipo Ax (cálido sub húmedo), Aw (cálido con lluvias de verano), Bs (seco), Bw (muy seco) (Granados Sánchez et al., 2013; Martínez Natarén et al., 2014) y C (frío). En estas áreas, la vegetación acompañante es variable, arbustiva, matorral rosetófilo, subinerme o espinoso (Castillo, 1991; Olhagaray et al., 2005; Treviño y Valiente, 2005; Villavicencio Gutiérrez, 2010; Granados Sánchez et al., 2013). 
Los sitios donde se establecen las poblaciones naturales tienen precipitación anual que va desde los 75 hasta los $850 \mathrm{~mm}$ anuales (Granados Sánchez et al., 2011), distribuida en el verano (Granados Sánchez et al., 2013), temporada en que ocurre el crecimiento mayor, desarrollo y floración en esta especie (Castillo, 1991; Ocampo Velázquez et al., 2009; Villavicencio Gutiérrez, 2010). Con la finalidad de establecer los sitios donde se reporta la especie, se realizan varios mapas sobre la distribución del orégano L. graveolens en territorio mexicano, apoyados con sistemas de información geográfica (SIG), entre ellos Granados Sánchez et al. (2013), que muestra un área amplia de distribución en el centro-norte del país y áreas pequeñas en el resto del territorio mexicano.

Dado que el orégano mexicano (L. graveolens) no está reportado para algunas áreas del país, la hipótesis es si a partir de sitios conocidos se pueden identificar los sitios y sus condiciones y a partir de ellos estimar la presencia para esta especie en otras áreas. Para corroborar esto fue necesario elaborar mapas para localizar áreas reales y potenciales donde se encuentra la especie reportada y los resultados obtenidos permitan proyectar condiciones de conservación, manejo, explotación y diversidad que ayuden a corroborar la identidad y variabilidad de las poblaciones que cubren el territorio mexicano.

\section{MATERIALES Y MÉTODOS}

En este estudio se consultaron bases de datos disponibles en Global Biodiversity Information Facility (GBIF, s. f.) con reportes de presencia de Lippia graveolens HBK, L. berlandieri y L. alba. Para el análisis, se emplearon los datos que tuvieron información con sitios georreferenciados. Entre las bases de datos consultadas se encuentra el Missouri Botanical Garden, Museo Nacional de Costa Rica, The New York Botanical Garden, los registros de la Comisión Nacional para la Biodiversidad (CONABIO), que se nutre de la información de herbarios como el del Instituto de Ecología, A. C., de la Universidad de Sonora, del Centro de Investigaciones Biológicas del Noroeste (CIBNOR), del Instituto de Botánica de la UNAM, así como del Centro Interdisciplinario de Investigación y Desarrollo Integral Regional unidad Durango (CIIDIR) del Instituto Politécnico Nacional.

Para la generación del modelo se utilizó el programa Maxent versión 3.3.3k (Phillips et al., 2006), para lo cual se realizó una matriz donde se determinó el uso de 17 parámetros climáticos (temperaturas y precipitaciones), dos fisiográficos (ecorregión y aridez) y uno geográfico (altitud). Este programa implementa un algoritmo de máxima entropía, mismo que genera una distribución de probabilidad sobre los puntos que conforman el territorio. Para ello, se calcula como el logaritmo del número de celda en la matriz menos el promedio de las probabilidades logarítmicas negativas de las localidades usadas para ajustar el modelo. Con la información anterior se generó una tasa de omisión que usa los registros de presencia, como los de prueba en función de un umbral acumulativo. Estos datos se utilizan para predecir la distribución de la especie.

RESULTADOS

En la consulta a las bases de datos con registros de $L$. graveolens se detectaron 505 puntos de referencia a partir de los cuales se recuperaron los que están georreferenciados. En total, de este último grupo se eligieron 350 puntos, con los cuales se realizaron los análisis posteriores. Los datos se anexan como material complementario de este manuscrito. Para el caso de L. alba y L. berlandieri hay 185 registros, de los cuales 120 están georreferenciados.

La Figura 1 muestra que L. berlandieri y L. alba presentan la misma distribución; al analizar los datos de las dos especies y traslaparlos son iguales. Al graficar los sitios registrados que tienen presencia de las

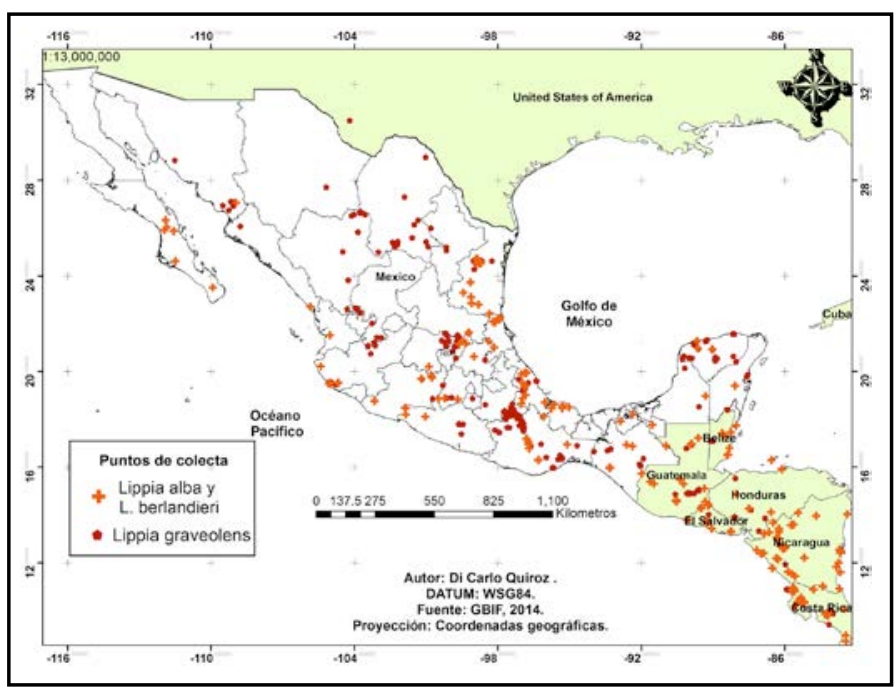

Figura 1. Sitios georreferenciados donde se reporta la presencia de L. graveolens, L. alva y L. berlandieri. Mapa elaborado por Jesús Di Carlo Quiroz Velásquez. 
dos especies se observa que tienen una distribución en forma de U, que corresponde al tipo de distribución neotropical (Figura 1), con presencia alta en sitios caliente-húmedos al sur del Eje Neovolcánico. Por su parte, L. graveolens tiene presencia en lugares desérticos del norte, centro y sur del país. En las áreas geográficas descritas se encuentran llanuras desérticas y sistemas montañosos que albergan a las especies mencionadas en altitudes y condiciones geográficas muy diversas. Esta especie no está restringida a la distribución tipo neotropical, pues en el centro de México se distribuye hacia el centro norte del país.

Los parámetros que se consideraron para la elaboración del mapa probabilístico de distribución de L. graveolens se muestran en la Tabla 1. En la columna de contribución se observa que la variable explicativa con mayor contribución es el porcentaje de lluvia del mes de junio; sigue en importancia la temperatura máxima promedio del trimestre más frío del año; el tercer factor de importancia es el porcentaje del promedio de lluvia del mes de marzo. La participación del factor ecorregión tiene una importancia inferior a $10 \%$, por lo que se considera importante pero con menor efecto. El factor altitud del sitio donde está el punto georreferenciado de la presencia de $L$. graveolens no tiene injerencia en la distribución de la especie, ya que en las interacciones su participación es de 0.8. De igual manera, el índice de aridez presentó un valor de cero.
En la Tabla 1 se muestra la columna de permutación, en que las variables tienen diferente valor a los expresados anteriormente. Por tanto, los valores en esta sección permiten dilucidar que la distribución de la especie está determinada principalmente por la temperatura del mes más cálido $\left(30.7^{\circ} \mathrm{C}\right)$, sigue en importancia la temperatura promedio de las mínimas registradas en el año (21 $\left.{ }^{\circ} \mathrm{C}\right)$. El tercer factor que interviene en la distribución de esta especie es la altitud (11.9 m.s.n.m.) que, aunada al promedio de lluvia del mes de junio (8.4\%), contribuyeron a delimitar la distribución de $L$. graveolens en México.

Mediante el programa Mexent se elaboró un mapa de distribución de L. graveolens (Figura 2) que muestra las zonas de acuerdo a los modelos con mejores condiciones pronosticadas. Por otra parte, en el mapa elaborado con la distribución probabilística de las áreas con presencia de L. graveolens en México y Centroamérica se distinguen los puntos que corresponden a sitios georreferenciados con la presencia de L. graveolens. Asimismo, en el mapa se aprecian las áreas que permiten estimar la presencia de la especie. La Figura 2 muestra la distribución probabilística de $L$. graveolens basada en los datos disponibles en GBIF, donde las cuatro variables (temperatura mínima del mes más cálido, temperatura mínima promedio anual, la lluvia y el porcentaje de lluvia de junio) contribuyen a determinar en $72 \%$ la presencia de la especie, donde se considera que ese nivel de explicación es alto y satisfactorio para este fin.

Tabla 1. Variables de los sitios con presencia de L. graveolens que están georreferenciados y contribuyen a la explicación de la distribución de la especie en el territorio mexicano

\begin{tabular}{|c|c|c|c|c|c|}
\hline Variable & $\begin{array}{c}\text { Contribución } \\
(\mathbf{\%})\end{array}$ & $\begin{array}{c}\text { Importancia de } \\
\text { permutación }\end{array}$ & Variable & $\begin{array}{c}\text { Contribución } \\
\text { (\%) }\end{array}$ & $\begin{array}{c}\text { Importancia dee } \\
\text { permutación }\end{array}$ \\
\hline \%Lluvia junio & 24 & 8.4 & PP_MAXCMS & 2.6 & 1.7 \\
\hline TMAXCMF & 19.6 & 2.2 & TMINCMC & 2.2 & 30.7 \\
\hline \%Lluvia marzo & 11.3 & 1 & TPROM & 1.4 & 0 \\
\hline Ecorregión & 8.8 & 4.6 & \%Lluvia dic & 0.9 & 0.3 \\
\hline \%Lluvia sep & 7.4 & 1.9 & Altitud & 0.8 & 11.9 \\
\hline TMIN_MIN & 4.4 & 21 & TMAX_MIN & 0.7 & 0.3 \\
\hline PP_PROM & 4 & 1.7 & TMAX_PROM & 0.6 & 1.7 \\
\hline PP_TOTAL & 3.7 & 6.5 & TMIN_MAX & 0.6 & 0.1 \\
\hline TMAX_MAX & 3.6 & 0.4 & TMIN_PROM & 0 & 0 \\
\hline PP_MINCMLL & 3.4 & 5.6 & Aridez & 0 & 0 \\
\hline
\end{tabular}




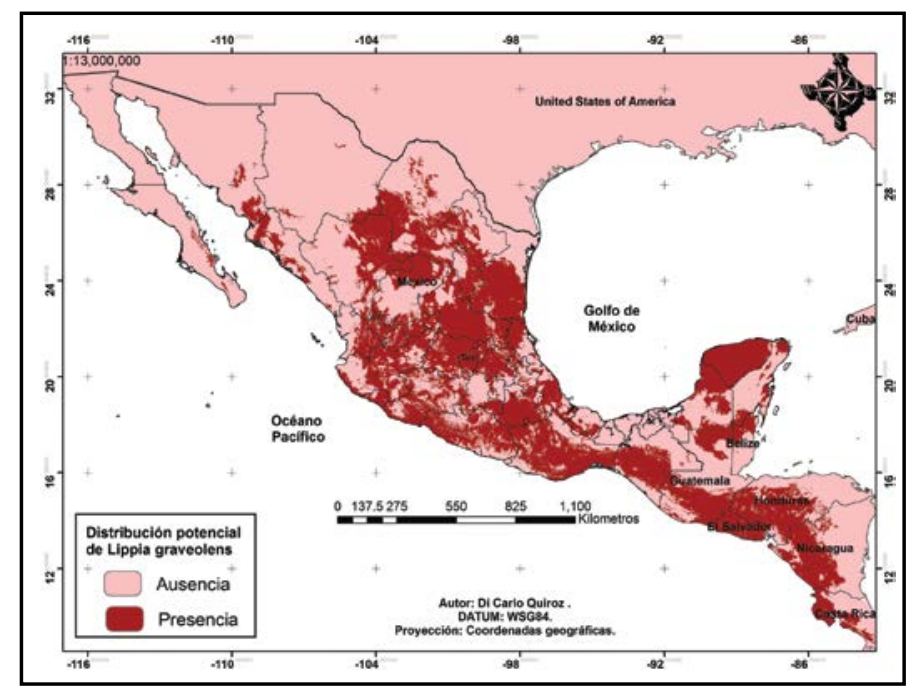

Figura 2. Mapa elaborado con la distribución probabilística de las áreas con presencia de L. graveolens en México y Centroamérica. Mapa elaborado por Jesús Di Carlo Quiroz Velásquez.

Los sitios donde se reporta la presencia de L. graveolens constituyen una zona muy amplia, diversa y dispersa en el territorio mexicano, por lo que algunos autores estiman que existen diferencias entre las poblaciones de la especie, que son propias de las adaptaciones a las particularidades de hábitat locales. A partir de esta información se sugiere realizar una cuadrícula más detallada con el objetivo de realizar muestreos que permitan hacer estudios moleculares y filogenéticos a fin de conocer la variabilidad intraespecífica de la especie, aspecto que se desconoce, pues solamente se han realizado estudios de este tipo a nivel intrapoblacional y se carece de los estudios interpoblacionales apoyados con estudios moleculares.

\section{DISCUSIÓN}

Reportes manejan como sinónimos a L. graveolens y a $L$. berlandieri o incluso a esta última, una subespecie $O$ variante de L. graveolens. Sin embargo, en la revisión que se realizó para este trabajo se consideraron diferentes (IPNI, 2014), sin que para ello exista una revisión de la taxonomía de la especie. Las sinonimias entre especies de $L$. spp. no son restrictivas para estas especies, por eso la importancia de este estudio de georreferenciar la especie de interés (Lippia spp.), pues hay otras plantas que se describen o mencionan con el mismo nombre (Deham et al., 2006; Meléndez Rentería et al., 2014). También la consulta de bases como la del GBIF ofrece la oportunidad de detectar errores de ubicación o registro de las muestras, como en el caso de un sitio con coordenadas 25.066 y 97.716 , el mapa lo ubica en India; sin embargo, al corregir, con 25.066 y -97.716 , los datos corresponden efectivamente a una localidad del municipio de San Fernando en Tamaulipas, México (GBIF, s. f.), como está descrita en la ficha técnica del material.

La distribución de L. graveolens es amplia en el territorio mexicano (Granados Sánchez et al., 2013; Martínez Natarén et al., 2014), donde está presente en suelos, climas, altitudes y tipos de vegetación muy variables (Castillo, 1991; Olhagaray et al., 2005; Treviño y Valiente, 2005; Villavicencio Gutiérrez, 2010; Granados Sánchez et al., 2013), por lo que este tipo de estudios además de ayudar a dimensionar las áreas de distribución de la especie (Alsos et al., 2009; Torres y Jayat, 2010) muestra una plasticidad fenotípica, ya que la planta se encuentra presente y se distribuye de manera natural en ambientes muy variados (Treviño y Valiente, 2005). Asimismo, dados los cambios derivados de la transformación, la pérdida de hábitat (Shipper et al., 2008) y el efecto del fenómeno del cambio climático y de invernadero, es importante conocer la distribución de la especie y las condiciones, áreas y espacios ocupados por la misma.

Por otra parte, el uso de modelos matemáticos para estimar el rango de distribución potencial es un elemento importante para la determinación de políticas de uso, manejo y conservación de especies como L. spp., que están sujetas a explotación en las áreas ocupadas (Hannah et al., 2002; Torres y Jayat, 2010). En este sentido, el programa Maxent está estructurado con base en algoritmos de máxima entropía y ofrece como resultado una distribución probabilística basada en la información conocida (Phillips et al., 2006), misma que se ha empleado en numerosos grupos taxonómicos, en áreas geográficas y condiciones diferentes (Treviño y Valiente, 2005.)

CONCLUSIONES

La información obtenida en los diversos bancos de información y herbarios disponibles mostró que a partir de las características del hábitat que ocupa L. graveolens se pueden estimar áreas potenciales de distribución de la especie. Esta información es de primordial importancia en cualquier estrategia de manejo, conservación o estudios taxonómicos, pues a partir del conocimiento de sus áreas naturales 
de distribución se pueden decretar políticas sobre manejo y explotación sustentable de estas especies. A partir de las características de los sitios georreferenciados se obtuvo un mapa probabilístico de distribución de las especies y los factores que al parecer son los determinantes de mayor importancia para la presencia de estas especies en México. Asimismo, se detectó que deben actualizarse con estudios in situ y así fortalecer las bases de datos existentes.

\section{LITERATURA CITADA}

- $\quad$ ALSOS, I. G. et al. Past and future range shifts and loss of diversity in dwarf willow (Salix herbacea L.) inferred from genetics, fossils and modeling. Global Ecology and Biogeography, 18(2): 223239, 2009.

- CASTILLO, Q. D. Distribución y ecología del orégano en el municipio de General Cepeda Coahuila. En R. MELÉNDEZ GONZÁLEZ, S. A. ORTEGA RODRÍGUEZ, y R. PEÑA RESÉNDIZ (Eds.), Estado actual del conocimiento sobre el orégano en México. Bermejillo, Durango. México: Universidad Autónoma de Chapingo. Unidad Regional de Zonas Áridas, 1991.

- DEHAM, S. et al. Lippia integrifolia versus L. boliviana (Verbenaceae). Darwiniana, 44 (2): 363-374, 2006.

- GRANADOS SÁNCHEZ, D. et al. Ecología, aprovechamiento y comercialización del orégano (Lippia graveolens H. B. K.) en Mapimí, Durango. Revista Chapingo. Serie Ciencias Forestales y del Ambiente, XIX(2): 305-321, 2013.

- GRANADOS SÁNCHEZ, D. et al. Ecología de la vegetación del desierto chihuahuense. Revista Chapingo. Serie Ciencias Forestales y del Ambiente, XVII(número Especial): 111-130, 2011.

- HANNAH, L. et al. Climate change-integrated conservation strategies. Global Ecology and Biogeography, 11 (6): 485-495, 2002.

- MARTíNEZ NATARÉN, D. A. et al. Genetic diversity and genetic structure in wild populations of Mexican oregano (Lippia graveolens H. B. K.) and its relationship with the chemical composition of the essential oil. Plant Systematics and Evolution, 300(3): 535-547, 2014.

- MELÉNDEZ RENTERÍA, P. et al. Microbiological effect of fermented mexican oregano (Lippia berlandieri Shaver) waste. Waste and Biomass Valorization, 5(1): 57-63, 2014.

- OCAMPO VELÁZQUEZ, R. V. et al. Biología reproductiva del orégano mexicano (Lippia graveolens Kunth) en tres condiciones de aprovechamiento. Agrociencia, 43(5): 475482, 2009.
- Olhagaray, E. et al. Cuantificación de orégano (Lippia berlandieri Shaver) en diez localidades del municipio de Nazas, Durango, México. 2da Reunión Nacional sobre orégano. Salaices, Chihuahua, México: Centro de Investigación para los Recursos Naturales, 2005.

- PHILLIPS, S. J. et al. Maximum entropy modeling of species geographic distributions. Ecological Modeling, 190, 231-259, 2006.

- SEMARNAT (SECRETARÍA DE MEDIO AMBIENTE Y RECURSOS NATURALES). Evaluación del riesgo de extinción de Lippia graveolens de acuerdo al numeral 5.7 de la NOM-059SEMARNAT-2001 (pp. 91-120). México: Autor, 2007.

- SHIPPER, J. et al. The status of the world's land and marine mammals: diversity, threat and knowledge. Science, 322 (5899): 225-230, 2008

- TORRES, R. y JAYAT, J. P. Modelos predictivos de distribución para cuatro especies de mamíferos (Cingulata, Artiodactyla y Rodentia) típicas del Chaco en Argentina. Mastozoología Neotropical, 17(2): 335-352, 2010.

- TREVIÑO, J. y VALIENTE, A. La vegetación de Tamaulipas y sUs principales asociaciones vegetales. En L. BARRIENTOS, A. CORREA, J. V. HORTA y J. GARCÍA (Eds.), Biodiversidad Tamaulipeca I. Instituto Tecnológico de Cd Victoria. Dirección General de Educación Superior Tecnológica, 2005.

- VILLAVICENCIO GUTIÉRREZ, E. E. Metodología para determinar las existencias de orégano (Lippia graveolens H.B.K.) en rodales naturales de Parras de la Fuente, Coahuila. INIFAP. 29 pp., 2010.

\section{De páginas electrónicas}

- $\quad$ GBIF (GLOBAL BIODIVERSITY INFORMATION FACILITY). Free and open access to biodiversity data [Portal] s. f. Recuperado el 5 de diciembre de 2014, de www.gbif.org

- IPNI (THE INTERNATIONAL PLANT NAMES INDEX) [Portal]. Recuperado el 5 de diciembre de 2014, de www.ipni.org/ 